Original article

\title{
Identifying risk factors for progression to AIDS and mortality post-HIV infection using illness-death multistate model
}

\author{
Omid Hamidi ${ }^{\mathrm{a}}$, Leili Tapak ${ }^{\mathrm{b}, *}$, Jalal Poorolajal ${ }^{\mathrm{c}}$, Payam Amini ${ }^{\mathrm{d}}$ \\ a Department of Science, Hamadan University of Technology, Hamadan, 65155, Iran \\ ${ }^{\mathrm{b}}$ Modeling of Noncommunicable Diseases Research Center, Department of Biostatistics and Epidemiology, School of Public Health, Hamadan University of \\ Medical Sciences, Hamadan 65175-4171, Iran \\ ${ }^{\mathrm{c}}$ Research Center for Health Sciences and Department of Epidemiology, School of Public Health, Hamadan University of Medical Sciences, Hamadan, 65175- \\ 4171, Iran \\ d Department of Epidemiology and Reproductive Health, Reproductive Epidemiology Research Center, Royan Institute for Reproductive Biomedicine, ACECR, \\ Tehran, Iran
}

\section{A R T I C L E I N F O}

\section{Article history:}

Received 1 July 2017

Received in revised form 16 August 2017

Accepted 22 August 2017

Available online 1 September 2017

\section{Keywords:}

HIV/AIDS

Highly active antiretroviral therapy

Multi-state model

Survival analysis

Tuberculosis

\begin{abstract}
A B S T R A C T
Background: There has remained a need to better understanding of prognostic factors that affect the survival or risk in patients with human immunodeficiency virus (HIV)/acquired immunodeficiency syndrome (AIDS), particularly in developing countries. The aim of the present study aimed to identify the prognostic factors influencing AIDS progression in HIV positive patients in Hamadan province of Iran, using random survival forest in the presence of competing risks (death from causes not related to AIDS). This method considers all interactions between variables and their nonlinear effects.

Methods: A data set of 585 HIV-infected patients extracted from 1997 to 2011 was utilized. The effect of several prognostic factors on cumulative incidence function (probability) of AIDS progression and death were investigated.

Results: The used model indicated that using antiretroviral therapy tuberculosis co-infection are two top most important variables in predicting cumulative incidence function for AIDS progression in the presence of competing risks, respectively. The patients with tuberculosis had much higher predicted cumulative incidence probability. Predicted cumulative incidence probability of AIDS progression was also higher for mother to child mode of HIV transmission. Moreover, transmission type and gender were two top most important variables for the competing event. Men and those patients with IDUS transmission mode had higher predicted risk compared to others.

Conclusions: Considering nonlinear effects and interaction between variables, confection with tuberculosis was the most important variable in prediction of cumulative incidence probability of AIDS progression.
\end{abstract}

(C) 2017 INDIACLEN. Published by Elsevier, a division of RELX India, Pvt. Ltd. All rights reserved.

\section{Introduction}

The human immunodeficiency virus (HIV) is the first leading cause of death from infectious disease worldwide and it has remained as a major public health issue (1). Acquired immunodeficiency syndrome (AIDS), that is the final and most serious stage of HIV infection, leads to sever damages to the body immune system. ${ }^{1,2}$ About 78 million people have been infected with the HIV virus and about 35 million people have died of AIDS-related diseases since the beginning of the epidemic. ${ }^{3}$ The prevalence of

\footnotetext{
* Corresponding author.

E-mail addresses: omid_hamidi@hut.ac.ir (O. Hamidi), l.tapak@umsha.ac.ir (L. Tapak), poorolajal@yahoo.com (J. Poorolajal), p.amini@yahoo.com (P. Amini).
}

HIV/AIDS in the overall population of Iran is less than $1 \%$ which is surpassed about $5 \%$ in high risk groups like injecting drug users (IDUs). ${ }^{4}$ In Iran until August 2016, a total of 30,727 people with HIV +/AIDS has been identified which 6016 patients have died., Although, no functional cure is available for HIV infection at present, the advent of antiretroviral treatment (ART) has led to a declination in the HIV-related mortality which in turn has helped patients to return to a relatively healthy and productive lives. ${ }^{1,2,7}$ Art also slows down AIDS progression process in an HIV-infected person and prolongs the survival of the patients. ${ }^{8}$ On the other hand, treatment of the HIV/AIDS can be complicated through several prognostic factors like chronic pathologies associated with immunodeficiency, chronic viral and bacterial infections. ${ }^{1,2}$ There are good evidences that show life span can be prolonged and the quality of life can be improved significantly if the levels of HIV 
remain suppressed and the CD4 count remains high (above 200). ${ }^{2}$ On the contrary, co-infection of HIV with other opportunistic infections especially tuberculosis (TB) may result in an increase in the risk of mortality. ${ }^{1,2,9}$

In spite of a declination in the HIV-related mortality, better understanding of the prognostic factors affecting the survival of the HIV-positive patients is of great importance to improve their life expectancy, especially in developing countries. ${ }^{1}$ However, limited studies have been conducted regarding survival of HIVinfected patients in these countries, especially those in the Eastern Mediterranean Region., ${ }^{1,2,10}$

There can be found several clinical and epidemiological followup studies that survival of the patients is the eventual outcome of interest, whereas during the study period individuals may experience some intermediate events. ${ }^{11}$ To model the passage of subjects through states the usual approach is utilizing separate analyses for every single endpoint. ${ }^{11}$ Nevertheless, an important drawback and shortcoming of this approach is that it does not account for the relationships between different endpoints. ${ }^{12}$ To overcome this issue, multistate models can be utilized for this type of data. ${ }^{13}$ Constructing multistate models provide a relevant modeling framework as well as a comprehensive view of a disease process. Moreover, by using multistate models proportions of individuals who are in the various states at some special time in the future can be estimated and more efficient use of incomplete information can be made when only fairly short portions of individual's disease histories are available. ${ }^{14}$

In HIV/AIDS disease, the data related to the survival of the patients has a structure that appropriately fits into multistate models because there is an intermediate event (AIDS) for the patients and some of the patients die without getting AIDS suggesting that the time from HIV to death process should be modeled by an illness-death multi-state model (Fig. 1). This is an important point that has not been considered in most HIV/AIDS studies. ${ }^{1,2}$ However, as mentioned the results (the coefficients of the covariates and their standard errors so their related Pvalues) can be affected dramatically which is because of the fact that they may fail to show the relations between different types of events. ${ }^{15}$

Designing the effective intervention strategies aimed to increase the life expectancy of HIV-infected patients is feasible through collecting reliable information about their survival times and the potential risk factors. ${ }^{1,2,9}$ On the other hand, appropriate statistical models can be utilized to reliable identification of important prognostic factors which helps to improve prediction accuracy of patients' survival. Therefore, the present study aimed to estimate the effect of potential risk factors on survival of HIV/ AIDS patients based on an illness-death multi-state model.

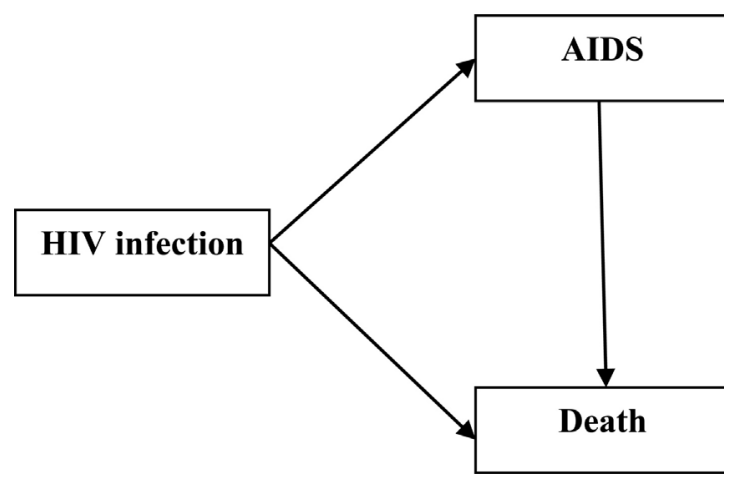

Fig. 1. Illness-death Model for HIV data.

\section{Materials and methods}

\subsection{Data description}

This retrospective cohort study was conducted in Hamadan, Iran, from 1997 to 2011 approved by the local Human Subject Review Board of Hamadan University of Medical Sciences. The number of 585 patients with HIV/AIDS was enrolled. The data was extracted from patients' medical records available at the triangular clinics (The triangular clinic is a kind of clinic established by the Iranian Ministry of Health and Medical Education and works for education, counseling, medical prevention and treatment in the three areas of consultation and treatment of addiction, consultation and treatment of AIDS and consultation and treatment of sexually transmitted diseases (other than AIDS). The services of these clinics are free of charge and they are performed anonymously. Therefore, patients need not introduce themselves or disclose their name and identity to use counseling).

The data collection was done using a predetermined checklist including following variables: age, gender, mode of HIV transmission (injection drug users [IDUs], sexual, mother to child, IDUs/ sexual, unknown), co-infection with TB, stage of HIV infection at diagnosis (asymptomatic/symptomatic/unclear), date of HIV diagnosis, date of progression to AIDS, date of death (if any), cause of death (if known) and receiving combination antiretroviral therapy (cART). All registered patients with a determined HIV infection were included in this study irrespective the age, gender, stage of the disease and date of diagnosis.

An HIV-infected case is defined as an individual with HIV infection regardless of clinical stage confirmed by laboratory criteria according to country definitions and requirements. ${ }^{2}$ In the Islamic Republic of Iran, the definitions of an HIV case and AIDS case are as follows respectively ${ }^{16,17}: 1$ ) an individual whose two sequential enzyme-linked immunosorbent assay tests were positive for HIV antibody followed and confirmed by a Western blot test; 2) an individual with a presumptive or definitive diagnosis of stage 3 or stage 4 condition and/or CD 4 count $<350$ per $\mathrm{mm} 3$ of blood in an HIV-infected subject.

There were three different outcomes of interest for investigation in this study: 1 ) the duration of time between diagnosis of HIV infection and progress to AIDS, 2) the duration of time between diagnosis of AIDS and occurrence of death from AIDS-related causes, and 3 ) the duration of time between diagnosis of HIV and occurrence of death from causes other than AIDS. Other patients who were lost to follow-up were considered as censored. The effect of several prognostic factors on the survival time to AIDS was investigated including gender, age, marital status, mode of HIV transmission, co-infection with TB and ART which is typically the name given to aggressive antiretroviral drugs used to suppress HIV viral replication and the progression of HIV to AIDS. Usually, it includes several drugs (typically three or four), taken in combination, such as two nucleoside reverse transcriptase inhibitors (NRTIs) and a protease inhibitor (PI), two NRTIs and a nonnucleoside reverse transcriptase inhibitor (NNRTI) or other such combinations. $^{2}$

\subsection{Data pre-processing and dealing with missing values}

Pre-processing of the data set was done in two stages: 1) fields with spelling errors, additional tokens, other irregularities and irrelevancies like outliers were corrected or removed; 2) Little MCAR test ${ }^{18}$ was performed to assess the missing completely at random (MCAR) mechanism for missingness $(p=0.953)$. So, the MCAR assumption did not reject and persons with at least one missing variable were removed from analysis. 


\subsubsection{Statistical model}

An HIV-infected patient may experience death without developing AIDS. This suggests that the HIV/AIDS process should be modeled by a multi-state statistical model. Fig. 1 shows a diagram of the illness-death process. Here we consider three transitions to two different states (AIDS and death): 1 ) HIV $\rightarrow$ AIDS, 2) AIDS $\rightarrow$ death, and 3) HIV $\rightarrow$ death. These events and transitions are modeled by a multi-state model with one absorbing state, death, and one non-absorbing (transient) state, AIDS. The model has two states. Interest lies in predicting the probability that a patient will be in one of the two states at some time after HIV infection as well as assessing the effects of prognostic factors on the transition intensities and survival rates. We utilized a commonly used multi-state model based on multivariable Cox transition intensities.

A multistate model is defined using a stochastic process of $(Y(t), t \in T)$ that have finite state space of $S=\{1, \ldots, p\}$ and $T=$ $[0, \tau]$ for a finite $\tau{ }^{13}$ This process has an initial distribution like $\pi_{\mathrm{h}}(0)=\operatorname{Pr}(\mathrm{Y}(0)=\mathrm{i}), \mathrm{i} \in \mathrm{S}$ and evolves over time and $\mathrm{F}_{\mathrm{t}-\text { which is a }}$ history (a $\sigma$-algebra) including of the observation of the process like the states previously visited and times of transitions are generated. ${ }^{13}$ Transition probabilities between two states of $h$ and $j$ states relative to the produced history, are defined as

$p_{i j}(s, t)=P\left(Y(t)=j \mid Y(s)=i, F_{s-}\right)$

for $i, j \in S, s, t \in T, s \leq t$ and transition intensities are defined as

$\alpha_{i j}(t)=\lim _{\Delta t \rightarrow 0} \frac{p_{i j(t, t+\Delta t)}}{\Delta t}$

In a multistate model, the insantaneous hazard of progression to state $j$ conditionally on occupying state $h$, can describe and characterize the multi-state process. ${ }^{15}$ "If $\alpha_{i j}(t)$ only depends on the history via the state $i=\mathrm{Y}(\mathrm{t})$ occupied at time $t$ then the process is Markovian". ${ }^{3}$

As the actual number of transitions and their orders are known, so a given observation covers a time priod between 0 and $\tau$ with $\mathrm{E}$ events. ${ }^{19}$ The process's start point is S0 at zero time and transition times will be $t_{\mathrm{j}}, j=1,2, \ldots, \mathrm{E}$ and process goes into $\mathrm{s}_{\mathrm{j}}, j=1,2, \ldots$, E. In order to estimate parameters of interest the following likelihood function is created ${ }^{19}$ :

$$
\left[\prod_{j=1}^{E} \alpha_{S_{j-1}}\left(t_{j}\right) \exp \left\{-\int_{t_{j-1}}^{t_{j}} \alpha_{S_{j-1}}(u) d u\right\}\right] \exp \left\{-\int_{t_{E}}^{\tau} \alpha_{S_{E}}(u) d u\right\}
$$

\subsubsection{Software}

All statistical analyses were performed at a significance level of 0.05 using the mstate library from the R software, version 3.3.1 (The R Foundation for Statistical Computing, Vienna, Austria; http://www.r-project.org).

\section{Results}

In order to fit illness-death multi-state model, we prepared the data and use a subset of the original data. Of the 582 HIV-positive subjects, used in the present study for analysis, 134 (23\%) patients developed AIDS, i.e. HIV $\rightarrow$ AIDS, (among them 45 (34\%) patients died, i.e. AIDS $\rightarrow$ death, 134 (23\%) patients died from causes other before developing AIDS, i.e. HIV $\rightarrow$ death and 314 (54\%) patients experienced no events (censored). The majority of the patients were male (88.95\%), single (45.42\%), aged $25-44$ years, not coinfected with TB and injecting drug users (81.51\%). The mean age at diagnosis was $32.59 \pm 8.71$ years, with a range from infancy to 66 years. The characteristics of the study population including gender,
Table 1

Characteristics of the study population infected with the HIV virus.

\begin{tabular}{|c|c|c|}
\hline Variables & Number & Percent \\
\hline \multicolumn{3}{|l|}{ Gender } \\
\hline Female & 515 & 88.95 \\
\hline Male & 64 & 11.05 \\
\hline \multicolumn{3}{|l|}{ Marital status } \\
\hline Single & 263 & 45.42 \\
\hline Married & 223 & 38.52 \\
\hline Divorced & 74 & 12.78 \\
\hline Widow & 19 & 3.28 \\
\hline \multicolumn{3}{|l|}{ Tuberculosis infection } \\
\hline No & 558 & 96.37 \\
\hline Yes & 21 & 3.63 \\
\hline \multicolumn{3}{|l|}{ Antiretroviral therapy } \\
\hline No & 11 & 8.21 \\
\hline Yes & 123 & 91.79 \\
\hline \multicolumn{3}{|l|}{ Modes of HIV transmission } \\
\hline Injecting drug users & 472 & 81.51 \\
\hline Sexual & 72 & 12.45 \\
\hline Mother to child & 9 & 1.55 \\
\hline Injecting drug users/Sexual & 26 & 4.49 \\
\hline \multicolumn{3}{|l|}{ Age group (year) } \\
\hline $1-24$ & 70 & 12.03 \\
\hline $25-44$ & 455 & 78.18 \\
\hline $45-74$ & 57 & 9.79 \\
\hline
\end{tabular}

age, marital status, mode of HIV transmission and co-infection with TB are given in Table 1.

Fig. 2, illustrates the cumulative hazards of transition from HIV to AIDS and death and AIDS to death for the patients, obtained from illness-death multistate model. In addition, stacked transition probabilities were plotted which is the most convenient way to interpret the probabilities. Here, the space between adjacent curves was filled with suitable colors (the darker one is more serious). Fig. 3 shows the result. The distance between two adjacent curves represents the probability of being in the

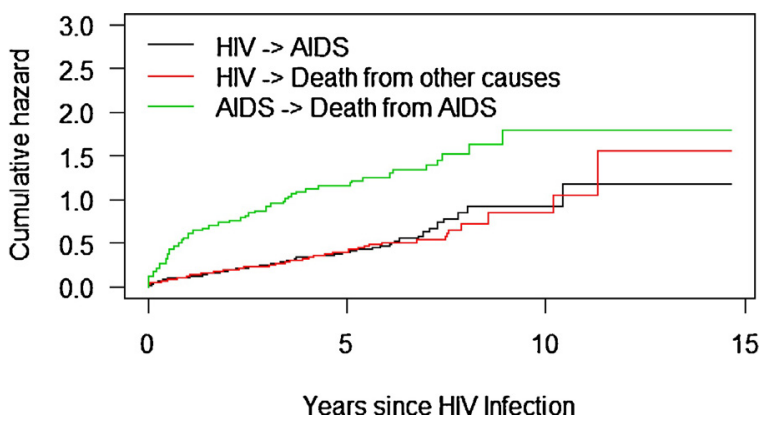

Fig. 2. Cumulative hazards from HIV to AIDS and death and AIDS to Death.

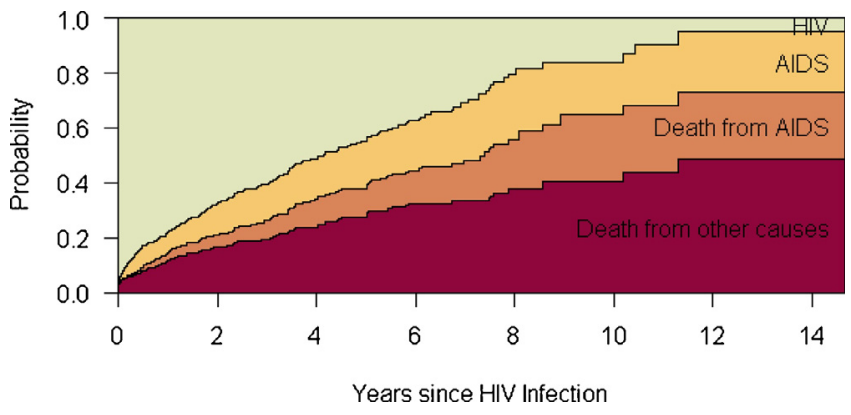

Fig. 3. Stacked transition probabilities. 
Table 2

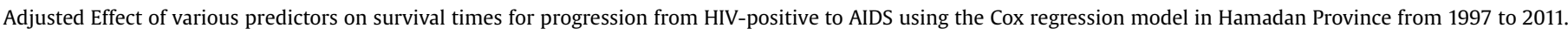

\begin{tabular}{|c|c|c|c|c|c|c|c|c|c|}
\hline \multirow[b]{3}{*}{ Covariates } & \multicolumn{9}{|c|}{ Transitions } \\
\hline & \multicolumn{3}{|c|}{$\mathrm{HIV} \rightarrow$ AIDS } & \multicolumn{3}{|c|}{ HIV $\rightarrow$ Death from other causes } & \multicolumn{3}{|c|}{ AIDS $\rightarrow$ Death from AIDS } \\
\hline & HR & $95 \% \mathrm{CI}$ & P-value & HR & $95 \% \mathrm{CI}$ & P-value & HR & $95 \% \mathrm{CI}$ & P-value \\
\hline Gender & & & & & & & & & \\
\hline Male & 1 & & & 1 & & & 1 & & \\
\hline Female & 1.20 & $(0.57,2.52)$ & 0.635 & 0.25 & $(0.05,1.31)$ & 0.099 & 0.33 & $(0.07,1.56)$ & 0.163 \\
\hline \multicolumn{10}{|l|}{ Marital status } \\
\hline Single & 1 & & & 1 & & & 1 & & \\
\hline Married & 0.93 & $(0.59,1.46)$ & 0.758 & 1.07 & $(0.62,1.41)$ & 0.730 & 2.02 & $(0.93,4.32)$ & 0.069 \\
\hline Divorced & 1.17 & $(0.67,2.01)$ & 0.585 & 1.23 & $(0.71,2.13)$ & 0.450 & 2.32 & $(0.89,6.05)$ & 0.083 \\
\hline Widow & 1.82 & $(0.78,4.23)$ & 0.164 & 0.93 & $(0.28,3.05)$ & 0.898 & 1.14 & $(0.13,10.33)$ & 0.903 \\
\hline \multicolumn{10}{|l|}{ Co-infection with TB } \\
\hline TB negative & 1 & & & 1 & & & 1 & & \\
\hline TB positive & 4.88 & $(2.88,8.32)$ & 0.001 & 0.44 & $(0.10,2.44)$ & 0.256 & 2.34 & $(1.13,4.83)$ & 0.023 \\
\hline \multicolumn{10}{|l|}{ Antiretroviral therapy } \\
\hline Yes & 1 & & & 1 & & & 1 & & \\
\hline No & 4.64 & $(2.27,9.49)$ & 0.001 & ND & - & - & 5.46 & $(2.23,13.41)$ & 0.001 \\
\hline \multicolumn{10}{|l|}{ Modes of HIV transmission } \\
\hline Injecting drug users & 1 & & & 1 & & & 1 & & \\
\hline Sexual & 1.21 & $(0.60,2.44)$ & 0.599 & 0.54 & $(0.19,1.56)$ & 0.255 & 0.88 & $(0.28,2.76)$ & 0.830 \\
\hline Mother to child & 16.8 & $(5.08,55.46)$ & 0.001 & 0.00 & ND & 0.993 & 1.05 & $(0.07,14.25)$ & 0.969 \\
\hline Injecting drug users/Sexual & 1.85 & $(0.92,3.76)$ & 0.087 & 0.38 & $(0.09,1.57)$ & 0.179 & 1.31 & $(0.36,4.68)$ & 0.682 \\
\hline \multicolumn{10}{|l|}{ Age group (year) } \\
\hline $1-24$ & 1 & & & 1 & & & 1 & & \\
\hline $25-44$ & 1.41 & $(0.72,2.73)$ & 0.311 & 1.03 & $(0.57,1.86)$ & 0.913 & 1.65 & $(0.21,13.21)$ & 0.635 \\
\hline $45-74$ & 2.36 & $(1.02,5.50)$ & 0.046 & 2.07 & $(0.99,4.33)$ & 0.054 & 1.20 & $(0.95,1.51)$ & 0.877 \\
\hline
\end{tabular}

HIV: human immunodeficiency virus; AIDS: acquired immunodeficiency syndrome; TB: tuberculosis; no data.

corresponding state. As can be seen, the probability of transition from HIV to AIDS and the probability of transition from AIDS to death increase over time.

Illness-death multistate model was fitted to time of transition from HIV to AIDS, HIV to death and AIDS to death. The effects of prognostic factors on hazards of transition to different states are shown in Table 2. The risk of AIDS progression (HIV $\rightarrow$ AIDS transition) was higher in patients who did not receive ART $(P=0.001)$ and in subjects who became infected through mother to child compared to IDUs $(\mathrm{P}=0.001)$ as well as in those who were coinfected with TB $(P=0.001)$. Besides, age had an increasing effect on the risk of progression to AIDS. So, the risk of AIDS progression was higher in the HIV positive individuals aged over 45 compared to individual aged $1-24$ years $(\mathrm{P}=0.046)$. The risk of transition from HIV to death (HIV $\rightarrow$ death) was lower in women compared to men (adjusted $\mathrm{HR}=0.13, \mathrm{P}=0.005$ and unadjusted $\mathrm{HR}=0.25$, $\mathrm{P}=0.099$ ). Moreover, the risk of $\mathrm{HIV} \rightarrow$ death transition in HIV positive individual aged over 45 to death was higher compared to individual aged 1-24 years (adjusted $\mathrm{HR}=2.85, \mathrm{P}=0.002$ and unadjusted $\mathrm{HR}=2.07, \mathrm{P}=0.054$ ). For transition between other states (AIDS $\rightarrow$ death), the risk of death was higher in AIDS patients co-infected with TB $(\mathrm{P}=0.023)$ and in patients who did not receive ART $(\mathrm{P}=0.001)$.

Fig. 2, illustrates the estimated cumulative hazards of transitions from HIV to AIDS and death (not related to death) as well as AIDS to death for the patients, obtained from illness-death multistate model. As seen, AIDS state has higher cumulative death hazards than HIV state in most time duration. Moreover, the cumulative hazard rate has a steeper slope in the first two years and after that slows down, suggesting that the hazard rate is not constant. However, after about the second year it is approximately linear that suggests a constant hazard rate. In addition for HIV state, the cumulative hazard rates for both transitions appear to be approximately linear over the study period, suggesting an approximately constant hazard rate. It is also seen that after 10 years the cumulative hazards of all states are coming closer which could be due to the small numbers of patients at 10 years and afterward.

In addition, stacked transition probabilities were plotted which is the most convenient way to interpret the probabilities. ${ }^{20}$ Here, the space between adjacent curves was filled with suitable colors (the darker one is more serious). Stacked prediction probabilities of being in initial state (HIV) as well as in the AIDS state were shown in Fig. 3. The distance between two adjacent curves represents the probability of being in the corresponding state. As can be seen, the probability of transition from HIV to AIDS and the probability of transition from AIDS to death increase over time. According to Fig. 3, the predicted transition probabilities at 5 years after being infected with HIV are about $20 \%$ to AIDS and about $25 \%$ to death not related to AIDS. Also, the predicted transition probability at 5 years after getting progressed to AIDS is about $10 \%$ to death related to AIDS. These probabilities increase to $23 \%, 0.44 \%$ and $25 \%$ for the last observation.

\section{Discussion}

Intermediate events play an important role in the disease courses in many survival studies. Utilizing separate analyses for each single endpoint (the usual approach in many studies) ${ }^{11}$ does not provide the possibility of uncovering relationships between different endpoints. ${ }^{12,13,21}$ Using multi-state models improves the understanding of variation in risk factors related to the evolution of diseases considerably 22 and constructing them allows for a comprehensive view of a disease process. They can use to obtain the probabilities and hazards of occurrence of different events. Multistate modeling framework makes allowances for estimation of proportions of individuals in the various states at different time points. They also uses of incomplete information more efficiently when there is only a short portion of individual's disease histories. ${ }^{14}$ Our study presented the effect of several predictors 
on the duration of time to three states in HIV-infected patients (the duration of time from HIV diagnosis to AIDS progression, the duration of time from AIDS initiation to AIDS-related death and the duration of time from HIV to death without AIDS progression) using illness-death multi-state models. We identified several risk factors strongly associated with survival times of transitions between different states of the disease (HIV $\rightarrow$ AIDS, HIV $\rightarrow$ Death and AIDS $\rightarrow$ Death).

Age was indicated to have a strong association with the risk of progression to AIDS (transition HIV $\rightarrow$ AIDS) and transition to death from causes not related to AIDS. According to the results obtained from multistate model, HIV positive subjects aged 45-74 years were at over two fold higher risk of AIDS compared to patients aged 1-24 years. Epidemiological studies indicated that patients over 50 years are at a higher risk of progression to AIDS compared to younger patients. $2,23-25$

According to our findings, the proportion of progression to AIDS was higher in women compared with men. On the other hand, the hazards of transition from AIDS to death and HIV to death were lower in women than in men. The difference between the two genders may be explain by two reasons: 1 ) the small sample size of women which can result in the difference to be just due to random errors; 2) the higher proportion of censorship in men compared with women ( $25 \%$ vs. $3 \%$, respectively). Therefore, the difference between two genders may only be attributed to more censorship that happened among men and small sample size of women. Therefore, interpretation of the hazard ratios between men and women must be done with caution.

TB is a leading preventable killer of people living with HIV infection. ${ }^{26}$ Our findings indicated that HIV positive subjects who were co-infected with TB had approximately five-fold higher risk of progression to AIDS compared to those who infected with HIV alone and subjects with TB coinfection who progressed AIDS had more than two-fold higher risk of death (2.33 times) compared to those without TB. This finding is in concordance with the results of other epidemiological studies., ${ }^{1,27}$ For example, Lopez-Gatell et al. reported that the hazard ratio of AIDS-related death was 2.4 times greater for patients co-infected with TB compared to those without TB. ${ }^{27}$ Therefore, the importance of treatment of TB in HIV infected people is revealed by this evidence. In 2004, an interim policy on collaborative TB/HIV activities and emphasized on three distinct objectives was published by the World Health Organization (WHO): (a) establishing and strengthening mechanisms for integrated delivery of TB and HIV services; (b) reducing the burden of TB among people living with HIV and initiating early antiretroviral therapy and (c) reducing the burden of HIV among people with presumptive TB and diagnosed TB. ${ }^{1}$

It was also shown that ART plays an important role in the risk of progressions from HIV infection state to AIDs state and AIDS to death. According to the results, HIV positive subjects not used antiretroviral therapy were at more than fourfold higher risk of AIDS progression compared to individual used that therapy. In addition not using antiretroviral therapy increases the risk of death in patients with AIDS considerably (about 5.5 times). According to the results of observational studies conducted in both resourcelimited and high-income countries, using ART leads to significant reductions in mortality rates with a range of $54-92 \%{ }^{28}$ Moreover, it has been indicated by evidence-based randomized clinical trials that early initiation of ART during antituberculosis treatment reduces mortality rates, especially in patients with profound immunosuppression. ${ }^{29,30}$ Effective treatment with ART helps to strengthening the immune system to regain the capacity to suppress infections and therefore controls replication of the virus within the body. ${ }^{1}$

According to the findings, most of the HIV infected subjects were male IDUs. One possible reason for the fact that the majority of HIV infections was transmitted through intra-venous drug use instead of sexual intercourse is that sexual contact with men or non-marital intercourse with women in Islamic Republic of Iran is strongly forbidden for men. ${ }^{1}$ Evidences have shown that the risk of HIV transmission among IDUs can be successfully reduced by access to sterile injecting equipment, methadone maintenance therapy and outreach services. ${ }^{1}$ In addition, the risk of AIDS progression was 17 times more in mother to child transmission compared with IDUs. This may be due to weak immune system of babies. The risk of mother to child transmission of HIV can be prevented by some strategies. For example, pregnant women with HIV can receive HIV medicines during pregnancy and in some situations; a woman with HIV may have a scheduled cesarean delivery (sometimes called a C-section). ${ }^{31}$ Moreover, because HIV can be transmitted in breast milk, mother to child transmission of HIV can be prevented by using safe and healthy alternatives of breast milk instead of breastfeed women's babies. ${ }^{31}$

There were a few limitations in the present study: 1) using data related to retrospective study recorded by registry centers that does not allow for verifying the accuracy of the data and can cause potential information bias ${ }^{1}$ while reliable sources of data obtained from prospective designs were required for survival analysis and associated prognostic factors ${ }^{1,2,9} ; 2$ ) considering the date of diagnosis as the beginning of the HIV infection and AIDS progression (some subjects may have become infected with HIV or progressed AIDS before they were diagnosed) that leads to underestimation/overestimation of the actual duration of transition times between two states; 3 ) the high proportion of censorship (here 54\%) may affect the model estimated proportion or probabilities that in turn might lead to transient state probabilities to be under-estimated or over-estimated. Several texts in multistate context investigated the asymptotic and distributional properties of the obtained estimates using counting processes theory ${ }^{32}$ that are asymptotically normal and unbiased. It is suggested to arrange several scenarios of simulate studies in multistate setting to investigate the sensitivity of the estimations numerically that would be an interesting idea for future work to investigate the impact of censoring rate on estimated parameters in different states of multistate models. There are also suggestions to handle high censoring rate as large as $90 \%$ in single point time to event data. ${ }^{33}$ It is also suggested to extend methods like fuzzy survival analysis to the multistate modeling context. The other issue was that since there was not sufficient information about receiving ART, we had to exclude this variable from our analysis because of the nature of multistate models. As it is well-established, ART can reduce the risk of AIDS progression as well as death. So, it is recommended to record information about taking ART in HIV positive patients progressed or not AIDS.

Despite these limitations, it was apparent the effect of several predictors on AIDS progression and death and AIDS-related deaths in a of a high-middle-income country which can provide useful information for institution of intervention measures to suppress the progression of HIV to AIDS and death and to reduce the risk of death among HIV-positive patients. ${ }^{1}$

\section{Conclusion}

The focus of the present study was to identify the important prognostic factors that affect the duration of time from HIV infection to AIDS and death and the duration of time from AIDS to death more efficiently using illness-death multi-state model. The results showed that several modifiable and non-modifiable predictors including co-infection with TB and using ART affect the risk of progression of AIDS and death in HIV-infected and AIDS patients. 


\section{Conflict of interest}

None.

\section{Acknowledgements}

We would like to appreciate the Vice-chancellor of Education for technical support and the Vice-chancellor of Research and Technology of Hamadan University of Medical Sciences for their approval and support of this work.

\section{References}

1. Mirzaei M, Poorolajal J, Khazaei S, et al. Survival rate of AIDS disease and mortality in HIV-infected patients in Hamadan, Iran: a registry-based retrospective cohort study (1997-2011). Int J STD \& AIDS. 2013; [0956462413486457].

2. Hamidi O, Poorolajal J, Sadeghifar M, et al. A comparative study of support vector machines and artificial neural networks for predicting precipitation in Iran. Theor Appl Climatol. 2015;119(3-4):723-731.

3. World Health Organization HIV/AIDS. Retrieved Jul y 6, 2016. 2016; Available from: http://www.unaids.org/en/resources/fact-sheet.

4. Haghdoost AA, Mostafavi E, Mirzazadeh A, et al. Modelling of HIV/AIDS in Iran up to 2014. J AIDS HIV Res. 2011;3(12):231-239.

5. Anvari-Tafti M, Eslami G, Teimourzadeh-Baboli A, et al. Food-Borne protozoan infection in HIV+/AIDS patients and healthy individuals: a case-control study in Iran. J Food Qual Hazards Control. 2016;3(3):93-96.

6. (NCAP)NCfAP. The Latest AIDS Statistics. [cited 201625 August]; Available from: http://www.aids.ir..

7. World Health Organization. HIV/AIDS. Retrieved August 4, 2014. (2013, July 2014); Available from: http://www.who.int/mediacentre/factsheets/fs360/en/

8. World Health Organization. HIV/AIDS. Retrieved February 2015. 2014, November 2014; Available from: http://www.who.int/mediacentre/ factsheets/fs360/en/.

9. Molaeipoor L, Poorolajal J, Mohraz M, et al. Predictors of tuberculosis and human immunodeficiency virus co-infection: a case-control study. Epidemiol Health. 2014;36:e2014024.

10. Tabarsi P, Chitsaz E, Moradi A, et al. Treatment outcome, mortality and their predictors among HIV-associated tuberculosis patients. Int J STD \& AIDS. 2012;23(9):e1-e4.

11. Andersen PK, Esbjerg S, Sørensen TI. Multi-state models for bleeding episodes and mortality in liver cirrhosis. Stat Med. 2000;19(4):587-599.

12. Eulenburg C, Schroeder J, Obi N, et al. A comprehensive multistate model analyzing associations of various risk factors with the course of breast cancer in a population-based cohort of breast cancer cases. Am J Epidemiol. 2016;183 (4):325-334.

13. Andersen PK, Keiding N. Multi-state models for event history analysis. Stat Methods Med Res. 2002;11(2):91-115.

14. Gentleman R, Lawless J, Lindsey J, et al. Multi-state Markov models for analysing incomplete disease history data with illustrations for hiv disease. Stat Med. 1994;13(8):805-821.
15. Beyersmann J, Latouche A, Buchholz A, et al. Simulating competing risks data in survival analysis. Stat Med. 2009;28(6):956-971.

16. Radfar S, Tayeri K, Namdari Tabar H. Practical Guidelines on How to Provide Consulting Services in Behavioral Disorders Centers. Tehran: Ministry of Health and Medical Education; 2009.

17. World Health Organization. WHO Case Definitions of HIV for Surveillance and Revised Clinical Staging and Immunological Classification of HIV-related Disease in Adults and Children. .

18. Little RJ. A test of missing completely at random for multivariate data with missing values. J Am Stat Assoc. 1988;83(404):1198-1202.

19. Hougaard P. Multi-state models: a review. Lifetime Data Anal. 1999;5(3):239264.

20. Lee J, Modi D, Jang $H$, et al. Multistate models on pleural effusion after allogeneic hematopoietic stem cell transplantation. Open Access Med Stat. 2017;7:15.

21. Hamidi O, Poorolajal J, Tapak L. Identifying predictors of progression to AIDS and mortality post-HIV infection using parametric multistate model. Epidemiol., Biostat Public Health. 2017.

22. Oliveira RdVCd, Shimakura SE, Campos DP, et al. Multi-state models for defining degrees of chronicity related to HIV-infected patient therapy adherence. Cadernos de Saúde Pública. 2013;29(4):801-811.

23. Bajpai RC, Raj P, Jha UM, et al. Demographic correlates of survival in adult HIV patients registered at ART centers in andhra pradesh, India : a retrospective cohort study. Public Health Res. 2014;4(1):31-38.

24. Tancredi MV, Waldman EA. Predictors of progression to AIDS after HIV infection diagnosis in the pre-and post-HAART eras in a Brazilian AIDS-free cohort. Trans R Soc Trop Med Hyg. 2014;108(7):408-414.

25. Walsh N, Mijch A, Watson K, et al. HIV treatment outcomes among people who inject drugs in Victoria, Australia. BMC Infect Dis. 2014;14(1):1.

26. Organization WH. WHO Policy on Collaborative TB/HIV Activities. Guidelines for National Programmes and Other Stakeholders. [Geneva, Switzerland, 2012. WHO/HTM/TB].

27. Lopez-Gatell H, Cole SR, Margolick JB, et al. Effect of tuberculosis on the survival of HIV-infected men in a country with low TB incidence. AIDS (London, England). 2008;22(14):1869.

28. Lawn SD, Kranzer K, Wood R. Antiretroviral therapy for control of the HIV associated tuberculosis epidemic in resource-limited settings. Clin Chest Med. 2009;30(4):685-699.

29. Blanc F-X, Sok T, Laureillard D, et al. Earlier versus later start of antiretroviral therapy in HIV-infected adults with tuberculosis. New Engl J Med. 2011;365 (16): 1471-1481.

30. Abdool Karim SS, Naidoo K, Grobler A, et al. Integration of antiretroviral therapy with tuberculosis treatment. New Engl J Med. 2011;365(16):14921501.

31. Kak L, Chitsike I, Luo C, et al. Prevention of Mother-to-child Transmission of HIV/ AIDS Programmes. Opportunities for Africa's Newborns: Practical Data, Policy and Programmatic Support for Newborn Care in Africa. .

32. Beyersmann J, Allignol A, Schumacher M. Competing Risks and Multistate Models with R: Springer Science \& Business Media.

33. Musavi S, Pokorny KL, Poorolajal J, et al. Fuzzy survival analysis of AIDS patients under ten years old in Hamadan-Iran. J Intel Fuzzy Syst. 2015;28 (3):1385-1392 